\title{
The role of GeneXpert in the diagnosis of Mycobacterium tuberculosis
}

\begin{abstract}
Introduction: GeneXpert (GX) is a novel, integrated, cartridge-based, nucleic acid amplification test with an established role for rapid diagnosis of Mycobacterium tuberculosis and detection of rifampicin resistance.

Aim: To evaluate the role of GX in pulmonary and extrapulmonary tuberculosis (TB) cases.

Material and methods: A prospective study was conducted in the pulmonary medicine department of a tertiary care hospital after the Ethics Comittee permission. Data of 257 presumptive TB patients was retrieved for GX, acid fast bacilli smear and culture (AFB smear and culture) and drug susceptibility test (DST). Sensitivity, specificity, positive predictive value (PPV), negative predictive value (NPV) of GX in diagnosis and determination of rifampicin resistance in pulmonary and extrapulmonary TB cases were calculated and compared with culture and DST results.

Results: Our study included 132 pulmonary and 125 extrapulmonary cases. On the basis of clinicoradiological and microbiological correlation, diagnosis of TB was confirmed in 104 pulmonary and 103 extrapulmonary cases. Out of a total of 104 pulmonary TB cases, 73 were rifampicin-sensitive and 31 were rifampicin-resistant cases. 103 extrapulmonary TB patients included 66 rifampicin-sensitive and 37 rifampicin-resistant cases. The sensitivity, specificity, PPV, NPV of GX in diagnosis and detection of rifampicin resistance in pulmonary TB was $95 \%, 93 \%, 98 \%, 84 \%$ and $96 \%, 100 \%, 100 \%, 96 \%$, respectively. The sensitivity, specificity, PPV, NPV of GX in diagnosis and detection of rifampicin resistance in extrapulmonary TB cases was $79 \%, 86 \%, 96 \%$, $47 \%$ and $97 \%, 95 \%, 97 \%, 95 \%$, respectively.

Conclusions: GX results are superior to smear microscopy and comparable to culture with shorter turnaround time.We recommend using it in routine TB diagnosis as this will expedite the management of patients with presumptive TB.
\end{abstract}

Key words: GeneXpert, presumptive TB cases, AFB culture

Adv Respir Med. 2020; 88: 184-188

\section{Introduction}

Tuberculosis remains a major health problem accounting for millions of new cases and deaths every year worldwide. Therefore, rapid detection of Mycobacterium tuberculosis (MTB) and rifampicin resistance in presumptive TB cases is essential for the early diagnosis and treatment, thereby reducing the risk of transmission of the disease, mortality rates and emergence of drug-resistant TB. AFB culture is considered as the gold standard test for final determination of TB but the turnaround time is $2-8$ weeks, and it requires trained personnel and expensive lab equipment [1]. Smear microscopy for acid fast bacilli (AFB) is one of the rapid and inexpensive tests available, but it has poor sensitivity and poor predictive value in the diagnosis of both pulmonary and extrapulmonary tuberculosis [2, 3]. Thus, rapid identification, which is essential for early treatment, improves patient outcomes, and more effective public health intervention relies on nucleic acid amplification techniques.

The GeneXpert MTB/RIF (rifampicin) assay is a novel, integrated, cartridge-based, nucleic acid amplification test (CBNAAT) for rapid diagnosis of MTB and quick detection of rifampicin resistance in both pulmonary and extrapulmonary

Address for correspondence: Jyotsna Joshi, Department of Pulmonary Medicine, Topiwala National Medical College \& BYL Nair Hospital, Mumbai, Maharashtra, India;

e-mail: drjoshijm@gmail.com

DOI: 10.5603/ARM.2020.0102

Received: 18.10.2019

Copyright (C) 2020 PTChP

ISSN 2451-4934 
samples [4-6]. GeneXpert (GX) test has been developed and launched by a foundation for innovative new diagnostics (FIND) and Cepheid Corporation in 2004. However, the development of the GeneXpert test was completed in 2008. The World Health Organisation (WHO) endorsed the GeneXpert for the use in TB endemic countries in December 2010 declaring it a major milestone for global diagnosis of tuberculosis [7].

The test isolates Mycobacterium genome from captured bacteria and amplifies DNA (deoxynucleic acid) using polymerised chain reaction. GeneXpert test identifies relevant 81bp (base pair) fragment of MTB rpoB gene using flourescent probes called molecular beacons. GeneXpert assay detects MTB by polymerise chain reaction (PCR) amplification of rpo gene and determination of rifampicin resistance by subsequent probing of this region for mutations that are associated with rifampicin resistance [8,9]. Turnaround time of the test is 90 mins. GeneXpert requires approximately 130 bacilli per $\mathrm{ml}$ of sputum for positive result, whereas Ultra test needs 16 bacilli per $\mathrm{ml}$ of sputum for the test to be positive. It is specific for MTB complex; i.e., it can differentiate MTB from other mycobacteria. For each specimen, the test is carried out in a closed system (cartridge), so there is a reduced risk of cross-contamination and human error.

Several studies have showed successful use of GeneXpert test on pulmonary and extrapulmonary samples with high sensitivity and specificity $[10,11]$. However, fewer false positive and false negative results of GeneXpert have also been reported recently, hence we decided to study and analyse its diagnostic accuracy in pulmonary and extrapulmonary TB.

\section{Material and methods}

We conducted a prospective study over a period of 2 years (1/9/2015-1/9/2017) in the pulmonary medicine department of a tertiary care hospital after the Ethics Comittee permission. Data of 257 consecutive presumptive TB patients (pulmonary and extrapulmonary) was retrieved for GeneXpert, AFB culture and drug susceptibility test (DST). Demographic data, clinical history, examination findings and radiological tests of these patients were also noted. Extrapulmonary cases included in our study were lymph node TB cases, TB pleural effusion cases, TB spine cases, other bone TB cases and TB meningitis cases. Presumptive TB pleural effusion cases were subjected to closed needle pleural biopsy and pleural biopsy samples were analysed for GeneXpert and AFB smear and culture tests. All cases involved in the study had clinical and radiological signs consistent with TB. Diagnosis of TB was made with the help of clinicoradiological correlation and microbiological tests positivity. Patients having no or incomplete data were excluded from the study. Sensitivity, specificity, negative predictive value and positive predictive value of GeneXpert in diagnosis and determination of rifampicin resistance in pulmonary and extrapulmonary TB cases were calculated by comparing it with culture and DST results. AFB culture method utilised for the study was MGIT liquid culture test, and the DST used was liquid culture DST.

\section{Statistics}

The sensitivity, specificity, positive predictive value and negative predictive value of GeneXpert were calculated according to the following formula.

Sensitivity: (true positive / true positive + false negative) $\times 100$ implies the test is positive when actually disease is present. Specificity: (true negative / true negative + false positive) $\times 100$ implies the test is negative when actually disease is absent.

Positive predictive value (PPV): (true positive/ /true positive + false positive) $\times 100$ calculates the probability that disease is present when the test is positive.

Negative predictive value:(true negative/ /true negative + false negative) $\times 100$ calculates the probability that disease is absent when the test is negative.

By applying the above mentioned formulas to the contingency tables, the sensitivity, specificity, PPV and NPV of GeneXpert in the diagnosis and detection of rifampicin resistance in pulmonary and extrapulmonary TB cases were calculated.

\section{Results}

Our study enrolled 257 presumptive TB cases which included 132 pulmonary and 125 extrapulmonary presumptive TB cases. On the basis of clinicoradiological and microbiological correlation, diagnosis of TB was confirmed in 104 pulmonary and 103 extrapulmonary cases. Of the 103 extrapulmonary TB cases; 48 were lymph node TB cases, 31 were TB pleural effusion cases, 11 were TB spine cases, 9 were other bone TB cases and 4 were TB meningitis cases. Out of a total of 104 pulmonary TB cases, 73 were rifam- 
picin-sensitive and 31 were rifampicin-resistant cases. 103 extrapulmonary TB cases included 66 rifampicin-sensitive and 37 rifampicin-resistant cases.

The sensitivity, specificity, positive predictive value, negative predictive value of GeneXpert in diagnosis of pulmonary TB and detection of rifampicin resistance in pulmonary TB was $95 \%, 93 \%, 98 \%, 84 \%$ and $96 \%, 100 \%, 100 \%$, $96 \%$, respectively. The sensitivity, specificity, positive predictive value, negative predictive value of GeneXpert in diagnosis of extrapulmonary TB and detection of rifampicin resistance in extrapulmonary TB cases was 79\%, 86\%, 96\%, $47 \%$ and $97 \%$, 95\%, 97\%, 95\%, respectively. The sensitivity, specificity, positive predictive value, negative predictive value of GeneXpert in diagnosis and detection of rifampicin resistance in lymph node TB cases was 77\%, 80\%, 95\%, $42 \%$ and $100 \%$, 89\%, 94\%, 100\%, respectively. Sensitivity, specificity, positive predictive value, negative predictive value of GeneXpert in diagnosis and detection of rifampicin resistance in TB pleural effusion cases was 71\%, 100\%, 100\%, $53 \%$ and $86 \%, 100 \%, 100 \%, 91 \%$, respectively. Sensitivity, specificity, positive predictive value, negative predictive value of GeneXpert in diagnosis and detection of rifampicin resistance in TB spine cases was 100\%, 100\%, 100\%, 100\%, respectively. When compared to previous studies, sensitivity of GeneXpert in pleural effusion cases was higher in our study as pleural biopsy samples obtained through closed needle pleural biopsy were also subjected to analysis. Low sensitivity of GeneXpert in pleural fluid samples could be due to very low numbers of bacilli as in the majority of cases, the accumulation of pleural fluid results from hypersensitivity reaction to tuberculous antigen rather than direct invasion of the organism into the pleura. Sensitivity, specificity, positive predictive value, negative predictive value of GeneXpert in diagnosis and detection of rifampicin resistance in other bone TB cases was $100 \%, 100 \%, 90 \%, 100 \%$ and $100 \%, 100 \%$, $100 \%, 100 \%$, respectively. Sensitivity, specificity, positive predictive value, negative predictive value of GeneXpert in diagnosis and detection of rifampicin resistance in CNS TB cases was $75 \%, 100 \%, 100 \%, 100 \%$ and $100 \%, 100 \%, 100 \%$, $100 \%$, respectively.

GeneXpert test was diagnostic in $13 \mathrm{AFB}$ culture-negative extrapulmonary TB cases and 5 culture-negative pulmonary TB cases. However, the test was false negative in 5 pulmonary and 21 extrapulmonary cases. GeneXpert test was false positive in all 5 cases, out of which 2 were pulmonary and 3 were extrapulmonary cases. The sensitivity of AFB smear was lower than GeneXpert sensitivity in both pulmonary and extrapulmonary TB.

The 68 DR TB cases on further DST were diagnosed as 50 (24\%) MDR TB, 17 (8\%) PreX$\mathrm{DR}(\mathrm{FQ})$, and $1(0.4 \%)$ XDR TB cases. There were 6 lymph node, 8 pulmonary, 2 spine and 1 CNS PRE XDR(FQ) TB cases.

\section{Discussion}

TB is a curable disease if detected early and treated effectively, thus it is highly important to have efficient and cost-effective diagnostic tests. Early diagnosis and appropriate treatment of TB can help in improving cure rates, reducing transmission rates, morbidity and mortality. ZN smear microscopy carries high risk of false negative results due to low sensitivity and it doesn't help to discriminate between drug susceptible and drug-resistant strains of MTB [2, 3]. These are owing to poor sample quality coupled with a need for technical expertise. Meanwhile, culture being the gold standard test for detecting MTB, requires several weeks to yield results, and largely depends on well-equipped laboratory facilities and skilled technicians [1]. Nucleic-acid-amplification tests provide more timely and accurate diagnosis of $\mathrm{TB}$, thereby contributing to early initiation of TB treatment. GeneXpert test is an integrated fully automated nucleic-acid-amplification test that detects MTB and rifampicin resistance within 90 mins. GeneXpert is a promising test with high sensitivity, specificity and rapid turnaround time [4-6]. The test is highly efficacious in pulmonary TB cases when compared to extrapulmonary cases. In addition, GeneXpert test is likely to improve the diagnostic accuracy of TB at places where AFB culture or DST facilities are not available.

In our study, the performance of GeneXpert test was analysed with pulmonary and extrapulmonary samples. Overall sensitivity, specificity, positive predictive value, negative predictive value of GeneXpert in diagnosis and detection of rifampicin resistance in pulmonary TB cases was $95 \%, 93 \%, 98 \%, 84 \%$ and $96 \%, 100 \%, 100 \%$, $96 \%$, respectively. In extrapulmonary TB cases, sensitivity, specificity, positive predictive value, negative predictive value of GeneXpert in diagnosis and detection of rifampicin resistance cases was $79 \%, 86 \%, 96 \%, 47 \%$ and $97 \%, 95 \%, 97 \%$, 95\%, respectively. These results are consistent with Cochrane metaanalaysis. Previous studies 
of the GeneXpert test have reported sensitivities of 47 to $90 \%$ in smear-negative, culture-positive pulmonary tuberculosis cases and 98 to $100 \%$ in cases of smear-positive, culture-positive pulmonary tuberculosis, with the specificity of GeneXpert being $99 \%$ to $100 \%$ [2, 12].

In the study conducted by Boehme et al,. the sensitivity was $99.8 \%$ for smear and culture-positive cases and $90.2 \%$ for smear-negative, culture-positive cases [13]. The sensitivity and specificity of the GeneXpert test in smear- and culture-positive pulmonary specimens was $98 \%$ and $100 \%$, respectively, which was consistent with data of previous studies. For smear-negative pulmonary specimens, the sensitivity of the test was $88 \%$, which is higher than that of Armand et al., and specificity was $100 \%$ comparable with previous studies [14]. For extrapulmonary smear-positive samples, sensitivity and specificity of GeneXpert was $100 \%$, whereas for extrapulmonary smear-negative samples, sensitivity and specificity was $67 \%$ and $100 \%$, respectively. The sensitivity and specificity for detecting rifampicin resistance in pulmonary and extrapulmonary samples was 96\%, 100\% and 97\%, 95\%, respectively, with respect to culture as reference standard.

Diagnosing extrapulmonary TB infection is quite challenging when compared to pulmonary TB due to significant low numbers of the organisms that can be recovered in the extrapulmonary samples. In our study, we found that the GeneXpert test was more sensitive in detecting spine and bone TB cases than pleural effusion and lymph node TB cases. The detection rate of MTB among smear-negative non-respiratory specimens by the GeneXpert test varies between studies with sensitivity rate of 20-66\%. As per studies by Armand et al., Causse et al., Tortoli et al. and Boehme et al., GeneXpert sensitivity in lymph node samples using AFB culture as reference standard, ranged from 50\% to $100 \%$ [13-16]. Sensitivity and specificity of GeneXpert in lymph node TB cases was $83.1 \%$ and $93.6 \%$, respectively, according to Denkinger metaanalysis [17]. Our study results of GeneXpert sensitivity in lymph node TB cases were $77 \%$, which was comparable with previous studies.

Metaanalysis findings of Denkinger et al. revealed sensitivity and specificity of this test among pleural fluid to be $46 \%$ and $99 \%$, respectively [17]. Another metaanalysis findings of $\mathrm{E}$. Penz et al. suggested sensitivity amongst pleural fluid was $37 \%$ and specificity of $98 \%$ [18]. However, in our study, sensitivity and specificity of GeneXpert in pleural effusion cases were $71 \%$ and
100\%, respectively. According to Denkinger metaanalysis, sensitivity and specificity of GeneXpert in CNS TB was $81 \%$ and 98\%, respectively [17]. As per study done by Penz et al., sensitivity and specificity of GeneXpert in CNS TB was $69 \%$ and 97\%, respectively [18]. In our study, sensitivity and specificity of GeneXpert in CNS TB was $75 \%$ and $100 \%$, respectively, and was consistent with previous studies.

Sensitivity and specificity of GeneXpert in bone TB as per study conducted by Held M. was $95 \%$ and $96 \%$, respectively [19]. Our results in bone TB were consistent with previously reported data.

In routine practice, the GeneXpert test is faster when compared to culture [4-6]. The GeneXpert test was positive for 163 of 194 culture-positive and 19 of 64 culture-negative samples from tuberculosis cases. In our study, the sensitivity of the GeneXpert test was found to be higher as that of culture in pulmonary cases, whereas lower than AFB culture in extrapulmonary samples. There were 5 and 21 false negative cases of GeneXpert for pulmonary and extrapulmonary cases, respectively. For AFB culture test, there were 5 and 13 false negative results in pulmonary and extrapulmonary samples, respectively. GeneXpert test was positive for all except for one smear-positive specimen, but the smear was positive only for 88 of 181 the GeneXpert test M.TB detected specimens. The sensitivity of microscopy was $58 \%(62 / 101)$ for culture-positive pulmonary specimens and $24 \%$ (26/93) for culture-positive extrapulmonary specimens. Therefore, the sensitivity of the GeneXpert test which was as rapid as smear was much higher than that of smear.

Smear-negative TB cases pose a challenge for the TB control programmes as they are associated with delayed or failure of diagnosis, unnecessary initiation of empirical tuberculous therapy and high risk of transmission. Therefore, the use of GeneXpert test, especially in countries with high TB prevalance like India, will help in rapid diagnosis and better treatment outcomes of smearnegative TB cases.

In our study, GeneXpert test was false positive in two pulmonary cases and 3 extrapulmonary cases. False positivity of GeneXpert test results has been reported previously and occurs because of the presence of dead MTB in the test samples, particularly among previously treated patients. There are highly likely chances for such patients to receive avoidable anti-TB therapy. Hence, careful history taking with emphasis on previous treatment with anti-TB drugs is essential 
to prevent unnecessary treatment of such false positive cases.

Our study included 31 pulmonary and 37 extrapulmonary DR TB cases. The percentage of DR-TB (24\%) cases in our study was higher when compared to the results of previous researches [20-22] due to referral bias, and it does not indicate true prevalence of DRTB in the population.

GeneXpert is useful for rapid detection of TB and identification of rifampicin resistance, especially in a high prevelance country like India. The results are superior to smear microscopy and comparable to culture with shorter turnaround time. We recommend using it in routine TB diagnosis as this will help in early diagnosis and the management of patients with presumptive TB. The test results must always be confirmed by culture and further DST in clinically discordant and DRTB cases.

\section{Conflict of interest}

None declared.

\section{References:}

1. Luetkemeyer A, Firnhaber C, Kendall M, et al. Evaluation of xpert MTB/RIF versus AFB smear and culture to identify pulmonary tuberculosis in patients with suspected tuberculosis from low and higher prevalence settings. Clinical Infectious Diseases. 2016; 62(9): 1081-1088, doi: 10.1093/cid/ciw035.

2. Walusimbi S, Bwanga F, Costa ADe, et al. Meta-analysis to compare the accuracy of GeneXpert, MODS and the WHO 2007 algorithm for diagnosis of smear-negative pulmonary tuberculosis. BMC Infectious Diseases. 2013; 13(1), doi: 10.1186/1471-2334-13-507.

3. Opota O, Senn L, Prod'hom G, et al. Added value of molecular assay Xpert MTB/RIF compared to sputum smear microscopy to assess the risk of tuberculosis transmission in a low-prevalence country. Clinical Microbiology and Infection. 2016; 22(7): 613-619, doi: 10.1016/j.cmi.2016.04.010.

4. Lawn S, Nicol M. Xpert®MTB/RIF assay: development, evaluation and implementation of a new rapid molecular diagnostic for tuberculosis and rifampicin resistance. Future Microbiology. 2011; 6(9): 1067-1082, doi: 10.2217/fmb.11.84.

5. Chang K, Lu W, Wang J, et al. Rapid and effective diagnosis of tuberculosis and rifampicin resistance with Xpert MTB/ RIF assay: A meta-analysis. Journal of Infection. 2012; 64(6): 580-588, doi: 10.1016/j.jinf.2012.02.012.

6. Marlowe EM, Novak-Weekley SM, Cumpio J, et al. Evaluation of the cepheid xpert MTB/RIF assay for direct detection of mycobacterium tuberculosis complex in respiratory specimens. Journal of Clinical Microbiology. 2011; 49(4): 1621-1623, doi: 10.1128/jcm.02214-10.

7. WHO, Rapid implementation of Xpert MTB/RIF Diagnostic test, WHO, 2011. Available at http://Whqubdoc.who.int?Pub- lication/2011/9789241501569. [Last accessed on Nov 15th, 2017].

8. Hillemann D, Rusch-Gerdes S, Boehme C, et al. Rapid molecular detection of extrapulmonary tuberculosis by the automated genexpert MTB/RIF system. Journal of Clinical Microbiology. 2011; 49(4): 1202-1205, doi: 10.1128/jcm.02268-10.

9. Blakemore R, Story E, Helb D, et al. Evaluation of the analytical performance of the xpert MTB/RIF assay. Journal of Clinical Microbiology. 2010; 48(7): 2495-2501, doi: 10.1128/jcm.00128-10.

10. Maynard-Smith L, Larke N, Peters J, et al. Diagnostic accuracy of the Xpert MTB/RIF assay for extrapulmonary and pulmonary tuberculosis when testing non-respiratory samples: a systematic review. BMC Infectious Diseases. 2014; 14(1), doi: 10.1186/s12879-014-0709-7.

11. Sharma S, Kohli M, Yadav R, et al. Evaluating the diagnostic accuracy of Xpert MTB/RIF assay in pulmonary tuberculosis. PLOS ONE. 2015; 10(10): e0141011, doi: 10.1371/journal. pone.0141011.

12. Ramirez HLB, García-Clemente MM, Álvarez-Álvarez C, et al. Impact of the Xpert ${ }^{\circledR}$ MTB/RIF molecular test on the late diagnosis of pulmonary tuberculosis. The International Journal of Tuberculosis and Lung Disease. 2014; 18(4): 435-437, doi: 10.5588/ijtld.13.0747.

13. Boehme C, Nicol M, Nabeta P, et al. Feasibility, diagnostic accuracy, and effectiveness of decentralised use of the Xpert MTB/RIF test for diagnosis of tuberculosis and multidrug resistance: a multicentre implementation study. The Lancet. 2011; 377(9776): 1495-1505, doi: 10.1016/s0140-6736(11)60438-8.

14. Armand S, Vanhuls P, Delcroix G, et al. Comparison of the Xpert MTB/RIF Test with an IS6110-TaqMan Real-Time PCR Assay for Direct Detection of Mycobacterium tuberculosis in Respiratory and Nonrespiratory Specimens. Journal of Clinical Microbiology. 2011; 49(5): 1772-1776, doi: 10.1128/jcm.0215710.

15. Causse M, Ruiz P, Gutierrez-Aroca JB, et al. Comparison of two molecular methods for rapid diagnosis of extrapulmonary tuberculosis. Journal of Clinical Microbiology. 2011; 49(8): 3065-3067, doi: 10.1128/jcm.00491-11.

16. Tortoli E, Russo C, Piersimoni C, et al. Clinical validation of Xpert MTB/RIF for the diagnosis of extrapulmonary tuberculosis. European Respiratory Journal. 2012; 40(2): 442-447, doi: 10.1183/09031936.00176311.

17. Denkinger CM, Schumacher SG, Boehme CC, et al. Xpert MTB/RIF assay for the diagnosis of extrapulmonary tuberculosis: a systematic review and meta-analysis. European Respiratory Journal. 2014; 44(2): 435-446, doi: 10.1183/09031936.00007814.

18. Penz E, Boffa J, Roberts DJ, et al. Diagnostic accuracy of the Xpert ${ }^{\circledR}$ MTB/RIF assay for extra-pulmonary tuberculosis: a meta-analysis. The International Journal of Tuberculosis and Lung Disease. 2015; 19(3): 278-284, doi: 10.5588/ijtld.14.0262.

19. Held M, Laubscher M, Zar HJ, et al. GeneXpert polymerase chain reaction for spinal tuberculosis. The Bone \& Joint Journal. 2014; 96-B(10): 1366-1369, doi: 10.1302/0301-620x.96b10.34048.

20. Jain A, Dixit P, Prasad R. Pre-XDR \& XDR in MDR and ofloxacin and kanamycin resistance in non-MDR mycobacterium tuberculosis isolates. Tuberculosis. 2012; 92(5): 404-406, doi: 10.1016/j.tube.2012.05.010.

21. Kozińska M, Brzostek A, Krawiecka D, et al. MDR, pre-XDR and XDR drug-resistant tuberculosis in Poland in 2000-2009. Pneumonol Alergol Pol. 2011; 79: 278-87.

22. Jain A, Mondal R, Prasad R, et al. Prevalence of multidrug resistant Mycobacterium tuberculosis in Lucknow, Uttar Pradesh. Indian J Med Res. 2008; 128: 300-306. 